\title{
Quorum Sensing Interfering Strategies and Their Implications in the Management of Biofilm-Associated Bacterial Infections
}

Samman Munir ${ }^{1}$

https://orcid.org/0000-0002-6659-5735

\author{
Asad Ali Shah ${ }^{1}$ \\ https://orcid.org/0000-0002-1345-3151
}

Muhammad Shahid ${ }^{1}$

https://orcid.org/0000-0002-1317-1621

\author{
Irfan Manzoor ${ }^{1}$ \\ https://orcid.org/0000-0003-3850-2690
}

Bilal Aslam ${ }^{2}$

https://orcid.org/0000-0002-5568-5049

\author{
Muhammad Hidayat Rasool ${ }^{2}$ \\ https://orcid.org/0000-0002-6324-1798
}

\author{
Muhammad Saeed ${ }^{2}$ \\ https://orcid.org/0000-0001-5011-5875 \\ Sultan Ayaz $^{3}$ \\ https://orcid.org/0000-0001-9551-5784 \\ Mohsin Khurshid ${ }^{\star}$ \\ https://orcid.org/0000-0002-3196-2857
}

\begin{abstract}
${ }^{1}$ Government College University Faisalabad, Department of Bioinformatics and Biotechnology, Faisalabad, Pakistan; ${ }^{2}$ Government College University Faisalabad, Department of Microbiology, Faisalabad, Pakistan; ${ }^{3}$ Government College University Faisalabad, Department of Eastern Medicine, Faisalabad, Pakistan.
\end{abstract}

Received: 2019.09.24; Accepted: 2020.06.01.

*Correspondence: mohsin.mic@gmail.com; mohsinkhurshid@gcuf.edu.pk; Tel.: +92-333-4301513

\section{HIGHLIGHTS}

- Quorum sensing (QS) regulates the development of biofilm formation and its dispersion.

- Targeting QS is a potential functional alternative to antibiotics

- The QS inhibitors can be utilized to impede the virulence in bacterial pathogens

- QS inhibitors could be very useful for the development of anti-biofilm pharmaceuticals.

Abstract: The bacterial species employ various types of molecular communication systems recognized as quorum sensing for the synchronization of differential gene expression to regulate virulence traits and biofilm formation. A variety of quorum sensing inhibitors; molecules that interfere with quorum sensing among bacteria have been examined which can block the action of autoinducers. Moreover, the studies have scrutinized various enzymes for their quorum quenching activity resulting in the degradation of signaling molecules or blocking of gene expression. So far, the studies have found that these approaches are not only capable to reduce the pathogenicity and biofilm formation but also resulted in increased bacterial susceptibility to antibiotics and bacteriophages. The effectiveness of these strategies has been validated in different animal models and it seems that these practices will be transformed in near future to develop the medical devices including catheters, implants, and dressings for the prevention of bacterial infections. Although many of these approaches are still in the research stage, the increasing library of quorum quenching 
Munir, S.; et al.

molecules and enzymes will open innovative perspectives for the development of antibacterial approaches which will extend the therapeutic arsenal against the pathogenic bacterial species.

Keywords: quorum sensing; interference; autoinducers; receptors; signaling.

\section{INTRODUCTION}

Quorum sensing is recognized as a mechanism of communication between bacterial communities and to collectively acclimate their behavior under the cellular density and their immediate environment. These mechanisms are advantageous for the entire community particularly at a higher cell density for example synthesis of virulence factors, the formation of biofilms, production of siderophores, and proteases [1]. The phenomenon of quorum sensing comprises the production of small molecules i.e. autoinducers (Als) which are released in proportion to cell density [2]. These autoinducing peptides were extensively studied and found to induce quorum sensing among Gram-positive bacteria and are species and strain-specific and have been reported particularly in Staphylococci, Enterococci, and Clostridium species. The acyl-homoserine lactones (AHLs) are another class of autoinducers that are widely reported in many Gram-negative bacteria particularly in Pseudomonas, Burkholderia, and, Acinetobacter species. Many other diverse types of signaling molecules including fatty acids, ketones, epinephrine, norepinephrine, Al-2, Al-3, or quinolones have been identified in bacterial species [1].

The phenomenon of quorum quenching is to interfere with quorum sensing which is majorly driven by the enzymes that are capable to degrade the $\mathrm{AHL}$ signals resulting in the disruption of the signals involved in quorum sensing. This interruption of communication between the bacterial communities can be accomplished by various processes. Firstly, the small molecules known as quorum sensing inhibitors can interfere with the production of autoinducers. Secondly, the quorum quenching macromolecules for example cyclodextrins and antibodies can scavenge the autoinducers. Moreover, the autoinducers can be hydrolyzed extracellularly by using enzymes [1]. Quite a few antagonist compounds have been identified among natural products or designed to quench for example polyphenols, ajoene, eugenol, 5-fluorouracil (5-FU), and azithromycin. The mechanisms of action of some of these quorum sensing inhibitors are unknown. Few of these for example azithromycin are mainly considered as antibiotics because of their ability to inhibit the growth of bacteria above a particular concentration [3].

The increasing antibiotic resistance has compelled the researchers to decipher newer therapeutic approaches [4-7]. The approaches to interfere with the quorum sensing can potentially inhibit the bacterial virulence and formation of biofilm that plays a key role in the emergence of antibiotic resistance. The quorum sensing triggers the biofilm formation which is composed of bacterial cells and an extracellular matrix consisting of proteins, polysaccharides, and DNA that may avert the penetration of antibiotics, therefore, inducing antibiotic tolerance. Moreover, the biofilm environments provide high cell density and increased selection pressure which ultimately increases the rate of development of resistant cells through genetic mutations or lateral gene transfer [1]. The elimination of biofilms, especially in the health care environment and devices, is a rising challenge, therefore, it is quite essential to develop alternative treatments in addition to conventional antimicrobial products. In this regard, the strategies which interfere with quorum sensing are increasingly being studied.

In this review, we have highlighted the latest findings related to the quorum sensing interfering methodologies and have summarized the potential of the quorum quenching molecules by the provision of data related to their efficacy in animal models as well as clinical trials for their successive medical applications.

\section{Quorum sensing: a bacterial crosstalk}

The different bacteria usually coexist forming multi-species communities within the host, for example, the oral cavity or the gastrointestinal tract. It is quite interesting that both gram-negative and gram-positive bacteria are capable of interacting with each other through the recognition and processing of signaling molecules among various species [8]. For instance, the biofilm is formed within the lungs of the infected patients suffering from cystic fibrosis mainly involving Burkholderia cepacia and Pseudomonas aeruginosa. It was reported that these two species utilized the acylated homoserine lactones (AHLs) autoinducer molecules for communication in a murine infection model. Here $B$. cepacia might recognize signal molecules processed by $P$. aeruginosa and not vice versa [9].

Further, it was indicated that eukaryotic cells can cross-talk with infectious bacteria and vice versa by using the signals. The Enterohemorrhagic Escherichia coli (EHEC); the causative agents of bloody diarrhea 
utilize the quorum sensing for cellular interaction. It was indicated that $E$. coli strains can interact with the host cell signaling molecules [10]. A knock-out mutant of EHEC that was not capable of synthesizing the autoinducer, was observed to react with the signaling molecules synthesized from the host which might activate the expression of the numerous virulence genes. Under other conditions, this might be possible that the signaling molecules processed via host may suppress quorum sensing systems of bacteria. Moreover, it is reported that the human respiratory epithelium can inhibit the cellular communication of $P$. aeruginosa. Furthermore, it is proposed that the mammalian respiratory system use this defense mechanism against the pathogenic organisms [11].

\section{Potential of quorum sensing as an antibacterial target}

Quorum sensing has also been evaluated as a promising antibacterial target as the strains having mutations within the essential quorum sensing genes were found to have reduced pathogenicity as observed in animal models. Quorum sensing systems in pathogenic microorganisms like $P$. aeruginosa remain active during human infections as seen in clinical trials where the signal molecules have been found in tissues or body fluids of infected patients [12]. As quorum sensing generally relies upon the interactions of various small-molecule ligands and receptors, it provides an explicit pharmacological pathway towards the inhibitor development. Whereas the biosynthetic pathway of signal molecules comprised a target for the pharmacologic inhibition, therefore, quorum sensing could also be inhibited through the prevention of pathogenic groups by the antibody-based sequestering or through the enzymatic inactivation of the signal molecules of quorum sensing [13].

At present, our knowledge about the capability of quorum sensing as the antibacterial target indicates that it is of great value among a few pathogenic organisms where it is obvious that disrupting the quorum sensing possesses a major effect on virulence [14]. In this case, clinically effective quorum sensing inhibitors are limited to the bacterial pathogens like $P$. aeruginosa and $S$. aureus [15]. The regulatory network of quorum sensing is more thoroughly studied in $P$. aeruginosa regulating biofilm maturation and various virulence determinants. Several $P$. aeruginosa quorum-sensing inhibitors have been characterized. Remarkably, several antibiotics comprising of ciprofloxacin, ceftazidime, and azithromycin have reported to hinder the quorum sensing at the sub-inhibitory concentrations [16]. The biofilm formation and quorum sensing interference to inhibit the surface colonization is shown in Figure 2.

\section{Quorum sensing and bacteriophages susceptibility}

Currently, the interests have risen significantly for the use of bacteriophages to treat the infections caused by multiple drug-resistant bacterial pathogens [17]. These phages are extremely abundant predators of bacteria on the earth and are increasingly being common for the treatment of bacterial infections. Though the bacteriophages exhibit an attractive way to evade the antibiotic resistance, bacteria also have acquired the mechanisms to respond to the phage attack. Foremost, the phage entry could be reduced by the production of the extracellular matrix or through the modification of the phage receptor expression or structure. Once within the cell, the phage DNA could be identified and destroyed by the adaptive inducible CRISPR-Cas system or the restriction enzymes [18].

The relationship among bacteriophage and quorum sensing was initially examined in $P$. aeruginosa however, in $E$. coli the capability of quorum sensing for regulating the defense mechanisms of phage was determined years later [19]. It was reported that $\mathrm{N}$ acyl-homoserine lactones caused a decrease in the adsorption rate by decreasing the number of receptors present on the surface of the cell. In V. cholerae, the lack of autoinducer synthase genes, and consequently in quorum sensing induction, reduces the resistance of phage that might be reestablished by adding the exogenous autoinducers, Al-2, CAl-1. The increase in phage resistance after the activation of quorum sensing was described through the downregulation of synthesis of $\mathrm{O}$-antigen that reduced the adsorption of bacteriophages and increased the production of hemagglutinin protease that was shown to inactivate the bacteriophages [20].

Taking into consideration, the use of quorum quenching compounds is an extremely promising technique to develop newer therapeutic applications. The use of these compounds in combination with phage therapy can enhance the sensitivity of bacteria towards phages through synergistic effects. Moreover, disrupting the quorum-sensing system of one species has shown to cause a decrease in the total biomass in multi-microbial cultures during the phage attack which leads to the fact that quorum quenching in conjunction with the phage therapy might be effective against the infections caused by multiple bacterial species [21]. 


\section{Antibiotics as QS inhibitors}

Since quorum sensing implies an overall change in the cell physiology and gene expression of bacteria, so the association between antibiotic tolerance and quorum sensing is multifactorial. For example, adding AHLs to the growing culture of $P$. aeruginosa has shown to enhance the number of bacterial persister cells after treating them with ciprofloxacin and carbenicillin [22]. Moreover, the transcriptional analysis in $P$. aeruginosa with the quorum sensing transcription regulator i.e., MvfR (PqsR) have shown that quorum sensing stimulates the expression of the peroxidases that protects the bacteria from the reactive oxygen species like hydrogen peroxide and beta-lactam antibiotics [23]. Further, the study involving the $P$. aeruginosa strain PAO1 and a regulator i.e. VqsM which is essential for quorum sensing induction was found to facilitate the antibiotic resistance by stimulating the expression of $n f x B$ gene; an antibiotic tolerant regulator conferring increased resistance to the tetracycline, kanamycin, and quinolones by regulating the expression of operon mexC-mexD-oprJ [24].

Even though several biochemical factors may be involved in the quorum-sensing mediated resistance to antibiotics, numerous studies have focused on the impact of biofilm formation in the antibiotic resistance among bacteria leading to treatment failure. These consequences have been examined within $P$. aeruginosa in animal models along with some other species like Staphylococcus aureus and Klebsiella pneumoniae [25]. Certain conditions in which the biofilm formation facilitates bacteria include defense mechanisms like alteration of gene expression, cellular physiological conditions, and physical barriers [26]. The las and $r h /$ quorum-sensing systems are necessary for the formation of biofilm and its disruption is associated with the high sensitivity to antimicrobial compounds as well as the host immune system among $P$. aeruginosa strains [11]. Furthermore, in $P$. aeruginosa an additional quorum sensing system called pqs system has shown mediating the programmed cell death causing the release of extracellular DNA that promotes antibiotic resistance and biofilm formation favoring the remaining cell population. In Acinetobacter baumannii, the antibiotics specifically the meropenem or levofloxacin has shown to stimulate the expression of an efflux pump that promotes the release of an $\mathrm{AHL}$ and therefore increases the quorum sensing mediated biofilm formation [27].

Considering the significance of quorum sensing in antibiotic resistance and biofilm formation, combination therapies using quorum quenching compounds were studied. In $P$. aeruginosa, the use of pharmacological compound i.e. benzamide benzimidazole and the inhibition of quorum sensing regulator MvfR restored the antibiotic susceptibility and reduced the biofilm formation [16]. Hamamelitannin and baicalin hydrate, a peptide-based quorum sensing inhibitor and the AHL targeting quorum sensing inhibitor increases the biofilm disruption in gram-positive bacteria e.g. S. aureus as well as in gram-negative bacteria like Burkholderia cepacia and $P$. aeruginosa and exhibited the synergistic effects in co-treatment with vancomycin, clindamycin, and tobramycin during in vivo and in vitro experiments [28]. The effectiveness of a large number of antimicrobial agents including the aminoglycosides, polypeptides, quinolones, glycopeptides, and cephalosporins, has been increased by adding the quorum sensing inhibitors [29, 30].

Combining all these findings indicates that the quorum-sensing inhibitors are promising agents to enhance antibiotic sensitivity and consequently reducing the massive doses of antibiotics. Moreover, related efficiency and trend have been examined with the antibiotic ciprofloxacin and lactonase enzyme for quorum quenching in mice model of infection [31]. Taken together, the quorum quenching, and antimicrobial agents possess encouraging effects, emphasizing that quorum quenching is an effective way of reducing the use of antibiotics and combat against the rising problem of antibiotic resistance.

\section{Antibodies as QS signal blockers:}

The control of quorum sensing using monoclonal antibodies seems to be an attractive approach due to its increased specificity and less cytotoxicity as seen in different studies. The antibody (RS2-1G9) was found to inhibit the 3-oxo-C12-HSL, therefore, decreasing the inflammation in the host during the Pseudomonas aeruginosa infection. Another antibody (XYD-11G2) catalyzed the hydrolysis of 3-oxo-C12-HSL signaling that resulted in decreased pyocyanin production among Gram-negative bacteria. The antibody (AP4-24H11) significantly attenuated the tissue necrosis in vivo using the infection model. Though the monoclonal antibodies have successfully blocked the QS signaling among pathogenic bacterial species, their therapeutic applications for various bacterial infections are yet to be confirmed in the clinical trials [32].

\section{Natural QS inhibitors}

The natural compound libraries can also serve as quorum quenchers or inhibitors. Particularly, plants are well known for producing highly complex compounds with a variety of therapeutic or medicinal properties. 
Though, the synthesis of various molecules is difficult, because of their complex chemical scaffolds; yet, the direct extraction method would be the best option to get these products. Enough compounds extracted from plants have already been found to have quorum inhibitor activity. Recently, it has been reported that the phenolic compounds extracted from plants can inhibit the biofilm formation in the PAO1 strain of $P$. aeruginosa when used in high concentrations [33]. However, these compounds have the opposite effect on the subinhibitory concentrations and supported biofilm growth. A few of these compounds include gallic acid, vanillin, and 4-hydroxybenzoic acid, which do not resemble the prototype of AHLs. Furthermore, derivatives of ellagic acid from the Terminalia chebula, also the aqueous extracts from Callistemon viminalis, Bucida buceras, and Conocarpus erectus have also been reported for the inhibition of QS of $P$. aeruginosa [34]. The derivatives of ellagic acid disrupt Rh1R and LasR, while simultaneously decreasing the production of virulence factors and increasing the sensitivity of the PAO1 strain of $P$. aeruginosa biofilm towards the tobramycin treatment. Another study was carried out among the Caenorhabditis elegans and found that the aqueous extracts have reduced the gut infection in $60 \%$ of nematodes and decreased the mortality rate by $50 \%$. Most importantly, all the extracts were useful with no toxic effects to the host and could be useful as therapeutic agents [34].

Apart from the extracted compounds from plant sources, various other natural compounds have also been derived from marine life and insects as well [35]. Considering the vast diversity of the natural world, a lot of beneficial compounds are still to be revealed, and researches into the uncharacterized and novel extracts would extend the list of useful compounds libraries.

\section{Inhibition of QS in animal models}

\section{Caenorhabditis elegans:}

The soil nematode worm $C$. elegans along with its associated bacterial species i.e. $P$. aeruginosa has been used as a model organism to figure out the efficiency of several quorum sensing inhibitors. Moreover, it is reported that the strains of $P$. aeruginosa that are capable of causing the disease in mice and humans are also able to kill the $C$. elegans worm [36]. Additionally, the pathogenesis of the $P$. aeruginosa strains in $C$. elegans is controlled with a similar cell density-dependent quorum sensing system as seen in human infections. Further, the sequencing of the $C$. elegans and $P$. aeruginosa genomes have made this association ideal for studying the disease biology. The ease of growth of $C$. elegans in the research laboratory is an additional advantage for its wide usage as a model organism to study the host defenses and pathogenesis in humans as well as animals. The nematode generally survives by feeding on the nontoxic bacteria; however, they are killed when feeding on the lawn of $P$. aeruginosa. The quorum sensing was found to regulate the hydrogen cyanide and phenazines production which has been associated with the killing of these worms. The wild-type $P$. aeruginosa has killed $100 \%$ worms while the quorum sensing knockout mutants killed only $10 \%$ of them which indicates the significance of quorum sensing in the virulence phenotype of the pathogen. The quorum-sensing inhibitors such as 4-NPO or garlic extract with the wild-type strains of $P$. aeruginosa were found to decrease the mortality rate of these worms to almost $40 \%$ and $5 \%$ [37]. The addition of the PvdQ acylase into the worms which were infected with the PAO1 strain of $P$. aeruginosa resulted in an enhanced lifespan and reduced the pathogenicity of the worms [38].

\section{Mice model:}

The mice were challenged with the $P$. aeruginosa embedded in the alginate beads that resulted in a chronic lung infection because of the bacteria caught in beads which seems like that of cystic fibrosis. Further, the bacterial $\mathrm{AHL}$ monitor having the quorum sensing regulated gfp gene implanted into beads resulted in the expression of the quorum-sensing controlled genes in the lungs. The quorum sensing inhibitor compounds inoculated with bacteria might be screened due to their capability to block or reduce the expression of gfp as the green fluorescent bacteria may be identified within lung tissues [39]. The extracts from Garlic were indicated to decrease the $P$. aeruginosa pathogenicity in mice when the quorum sensing defective $P$. aeruginosa mutant LasR was used in the burn mice model [40].

\section{Other models:}

Various models such as the rabbit osteomyelitis model, rabbit endophthalmitis model, and mouse septic arthritis model have been used to observe the role of accessory gene regulator in the pathogenesis of $S$. aureus [41]. The Drosophila melanogaster was used to study the quorum sensing and quorum sensing inhibitors and to study the dynamics of $P$. aeruginosa infection and the association between the pathogenicity and formation of biofilm in this bacterial species. The natural products were found to stimulate the organo- 
sulfur compounds such as S-phenyl-L-cysteine sulfoxide which has been indicated as the quorum sensing inhibitor which inhibited the $P$. aeruginosa infection, biofilm formation as well as quorum sensing [42].

\section{QS inhibitors in clinical trials}

The previously certified and commercialized quorum sensing inhibitors were implicated in the clinical trials, even though their basic uses and specialized biological activity shows no association with bacterial quorum sensing, although they were cytotoxic and possess antimicrobial activities and were bactericidal [43, 44]. The azithromycin has been widely used for the treatment of pulmonary transplanted and cystic fibrosis patients in clinical trials. This antibiotic enhanced the quality of life of the affected patients but was unable to reduce the bacterial load among infected patients [45]. Simultaneously, the capability of azithromycin to disrupt the bacterial signaling at non-bactericidal concentrations for $P$. aeruginosa strains was exhibited in vitro [46]. Further, the effect of azithromycin on the $P$. aeruginosa quorum-sensing among the patients with ventilator-associated pneumonia was determined [44]. These studies explained the anti-virulence impact of the azithromycin among the high-risk group patients, but the results obtained were not significant.

The extracts from Garlic are widely known for its quorum quenching activities and have been evaluated in a clinical trial for the treatment of patients suffering from cystic fibrosis, however, the results were unable to prove the therapeutic benefits of garlic in these patients [47]. The anti-cancer drug, 5-Fluorouracil which is a pyrimidine analog was shown to impede the quorum-sensing regulated virulence factors in $P$. aeruginosa as seen in-vitro experiments, therefore, its use as a coating for catheters was suggested in the clinical trials [43].

Eventually, quite a few numbers of quorum quenching molecules have reached up to the clinical trials however they tend to exhibit some valuable effects of quorum sensing inhibitors. Even though many pieces of evidence of the theory were implemented in the animal models, further work needs to be devoted to authenticating these methods in the clinical stages to verify its therapeutic application.

\section{QS inhibitors in medical devices}

Most of the hospital-acquired infections are linked with the use of various medical devices. Biofilm forming and multi-drug resistant bacteria are mostly responsible for hospital-acquired infections that cause serious medical complications, also a high risk of mortality and morbidity. Considering the capability of quorum quenching to inhibit the bacterial virulence, developing innovative medical devices by using quorum quenching is of utmost interest [48]. New generations of dressings, catheters, contact lenses, aerosols, trauma, and orthopedic devices or implantable devices are recently being developed [49-51]. The studies using quorum sensing inhibition for various medical devices are summarized in table 1.

Initially, a quorum-sensing inhibitor was studied for the functionalization of the catheters. The furanones have been covalently attached which are shown to reduce the formation of biofilm by Staphylococcus epidermidis reference strain and to control the infection (up to 65 days) within a sheep model [52]. For the coating of a central venous catheter, the 5-fluorouracil was used and was found effective and analogous to the traditionally used chlorhexidine and silver sulfadiazine coated catheters during a study comprising of 960 patients admitted in intensive care units in the USA [43]. Though the association with quorum sensing was not much appraised in the studies, the 5-fluorouracil coated catheters exhibited a decreased level of contamination especially the gram-negative bacteria, compared with the conventional coatings that suggest the interference of 5 -fluorouracil with the AHL dependent quorum sensing.

The coatings using polyethylene glycol comprising of quorum sensing inhibitor DHP(5-methylene-1(prop-2-enoyl)-4-(2-fluorophenyl) dihydropyrol-2-one) was indicated to decrease the colonization of $P$. aeruginosa strain MH602 and an S. aureus strain. The combinations of DHP derivatives and furanone and remained covalently attached on the surface of the glass which considerably decreased the adhesion of $P$. aeruginosa strain PAO1 and an S. aureus strain [53]. The delivery systems based on the varnishes, emitting quorum sensing inhibitor TZD-8 (thiazolidinedione-8) were used for the catheters and were found to be active against the biofilms produced by Candida albicans [54]. In a recent study, polyphenols from honey have been used into the scaffold of selenium nano-vectors for the quenching of $P$. aeruginosa strain PAO1 for in vivo and in vitro evaluation [55].

The inhibition of agr-based quorum sensing in $S$. aureus was accomplished using inhibiting peptides for various biomaterials. In a study, the macrocyclic peptides were inserted into the non-woven polymer nanofibers which were confirmed to maintain their biological activity against the $S$. aureus for three weeks [56]. The surface coating using click chemistry has been considered for coating of pro and anti-quorum sensing peptides i.e. AIP-I and TrAIP-II which were found significantly efficient for the inhibition of $S$. aureus biofilms [57]. The interaction of antibiotics with the quorum sensing inhibiting peptides FS3 was also 
determined using daptomycin and found quite effective in combination with the FS3-coated prosthesis against the $S$. aureus infections [58]. Likewise, the RNAIII-inhibiting peptide was found very effective inside a Dacron tube graft for decreasing infections associated with $S$. epidermidis strains [59].

The quorum sensing inhibiting materials have been obtained following the immobilization of peptides or QSIs, quorum quenching enzymes have also been extensively scrutinized as these acts on the secreted autoinducers and do not require direct contact with the microbial surface to disrupt the communication. Aspergillus melleus Acylase has been incorporated successfully within silicon catheters and polyurethane coatings decreasing the biofilm formation of $P$. aeruginosa ATCC10145 and PAO1 strain [60, 61]. The acylase in combination with $\alpha$-amylase obtained from the Bacillus amyloliquefaciens was found to delay the biofilm formation of $E$. coli and $P$. aeruginosa reference strains for almost 7 days in a rabbit model of infection [62]. The acylase from the porcine kidney immobilized onto the carboxylated polyaniline nanofibers (cPANFS) to produce Nano biocatalysts reduced the formation of biofilm by $P$. aeruginosa PAO1. The topical application using lactonases obtained from Bacillus species has also been examined in a mice model of burn infection using the $P$. aeruginosa reference strain PAO1. The gel containing lactonase stopped the systemic spread of bacteria, reduced mortality, and offered synergistic activity with ciprofloxacin in burn infections [31].

Since the stability of the enzyme is the main obstacle in developing the bio-based materials, the various enzymes from the extremophiles were also considered. Specifically, the PLL SSoPox enzyme from the Sulfolobus solfataricus has found to possess an exquisite mode of quenching bacterial virulence [49]. This highly stable enzyme was initially immobilized on the Nanoporous alumina membranes while maintaining the strong effect of decreasing the secretion of virulence factors, elastase, and pyocyanin of $P$. aeruginosa PAO1 $[63,64]$. SsoPox-W2631, a variant enzyme, has been exhibited to considerably decrease the virulence of $P$. aeruginosa of the 51 clinical isolates from the diabetic foot ulcerations by retaining its efficacy towards the bacterial strains after immobilization in the polyurethane coating through glutaraldehyde cross-linking [65]. These enzymes have also used for intratracheal administration which significantly increased the survival rate in rats as observed in a pneumonia infection model for a reference strain of $P$. aeruginosa [51]. Apart from the studies using the acyl-homoserine lactones-based quorum sensing quenchers, the latest study has explained the use of Al-2 synthesizing kinase LsrK. The enzyme was combined with the biological polymers i.e. alginate and chitosan and ATP were added which ultimately decreased the AI-2 mediated quorum sensing [66].

A recent study has measured the QS inhibition potential of various alkyl gallates used in food preservation including octyl gallate $(\mathrm{OG})$ and propyl gallate $(\mathrm{PG})$ and hexyl gallate $(\mathrm{HG})$ against $P$. aeruginosa. The QS gene expression experiment suggested that that the HG inhibited RhIR, whereas OG inhibited PqsR. The study highlighted that alkyl gallates great potential as a QS inhibitor against $P$. aeruginosa [67]. Quorum quenching based devices have got special attention as that they could potentially prevent hospital-acquired infections through effective control of biofilm formation and bacterial virulence. Still, more efforts are required to verify the concepts in vitro as well as in animal or clinical studies. The efficiency of these devices must be determined not only in bacterial strains model but also on phenotypically and genetically diverse clinical strains. Even though the development of medical devices looks less difficult compared to the drugs, the regulatory issues need to be addressed for authenticating the capability of these systems for their therapeutic applications. The wide range of quorum quenching enzymes and quorum sensing inhibitors and their medical applications will ultimately lead to the development of innovative medical devices.

\section{Quorum quenching; in silico approaches:}

The detection and discovery of enzyme inhibitors in medicinal chemistry are also supported by the computer-based methods for the exploration of anti-quorum sensing substances which helps to save the cost and time in performing experiments by preselection of the potential candidates through in silico screening.

A study conducted for the virtual screening to find the RhIR and LasR quorum sensing inhibitors within $P$. aeruginosa. Among the 1,920 compounds, docking detected five candidate binders for RhIR and LasR receptors. Their capability to inhibit the expression of the virulence factors i.e. elastase, hemolysin, and protease was further validated through in vitro experiments [68]. In another study, a library was screened comprising of 3,040 natural compounds for finding the new quorum-sensing inhibitors of the receptor Las $P$ from $P$. aeruginosa. Based upon the molecular docking results, the in vitro experimental processes were limited to just 22 auspicious candidate inhibitors. These compounds were further investigated to downregulate various virulence factors [69]. The structural compound library namely SuperDrug and SuperNatural which comprise of natural compounds were screened to find the inhibitors for the LasR receptor 
from $P$. aeruginosa. By employing molecular docking and virtual screening, few compounds were found capable to inhibit the quorum sensing signaling cascade [70].

A variant Boolean network modeling as an in silico multistep modeling approach to study the timedependent characteristics of the Pqs, Las, and $R h 1$ signaling systems in $P$. aeruginosa [71]. Their goal was to examine the metabolic and regulatory interaction between quorum sensing inhibitors, signaling molecules, receptor antagonism, and expression of various virulence factors e.g. rhamnolipids, pyocyanin, and elastase. During the simulation, they revealed that the signaling molecules PQS and $\mathrm{HHQ}$ are reduced when PqsBCD expression is suppressed by suitable inhibitors. This network approach also allowed them to anticipate the quantitative impact of PqsR antagonists and Pqs inhibitors. In S. aureus a crystal structure of AgrA LytTr domain was determined which was necessary to bind the DNA. Afterward, the virtual screening using a small library comprising of almost 500 compounds revealed three inhibitors that disrupt the AgrA binding to DNA [72].

\section{Quorum sensing interference using animal infection models; bottlenecks}

So far, several animal infection models have validated that the mutants which are deficient in quorum sensing are far less infectious than their parental strains; therefore, quorum sensing interference stimulates the potential of microbes by increasing the survival rate of host and resulted in a decrease in the bacterial counts and bacterial associated damage [73]. Few of the commonly used infection models include the nematodes like Caenorhabditis elegans, arthropods such as Galleria mellonela, zebrafish, and fruit fly which are informative and valued yet lacks the accurate human physiological features. Furthermore, the mice and immunocompetent individuals were also used as a model; however, it is understood that the $P$. aeruginosa is an opportunistic pathogen, therefore it was not clear that the immune response of the immunocompromised patients will be able to clear the bacterial infections following the therapeutic use of quorum sensing interference therapies. Besides affecting the immunocompromised individuals, the $P$. aeruginosa strains are the primary cause of the death in cystic fibrosis patients; therefore, various mice models exist that combine particular mutations in the membrane protein termed as cystic fibrosis transmembrane conductance regulator (CFTR) that could mimic several properties of the infection in humans. Nevertheless, cystic fibrosis is a quite complicated disease the number of reported mutations within cftr gene is much more (more than thousand) which are associated with this disease; thus, it seems to be challenging to develop a murine model which closely resemble the human disease [74].

Many of the modern models are appropriate for studying acute infections but very few are suitable for chronic infections i.e. cystic fibrosis. This particular model was used to conclude that quorum sensing interference by using azithromycin can impede with the alginate production by the mucoid strain NH57388A, therefore, reducing the damage to the host, still, was unable to considerably enhance the survival of mouse [75]. The thermally induced injury model is an alternative kind of model which is similar in a situation as observed in humans as it involves the production of second or third-degree burns on the dorsal side of the mouse using hot water followed by inoculation of burn. It was observed that quorum sensing deficient mutants e.g. lasl, lasR, pqsA, rh1l, and lasl rh1l were less virulent as compared to the parental strains [76]. Furthermore, it has also been employed to examine the inhibitory effect of anthranilic acid analogs including 4CABA, 6CABA, and 6FABA for the blockage of quinolone quorum-sensing signals. These compounds significantly decreased the mortality rate in the mouse at the appropriate doses. However, these compounds were assessed on a single dose, so it is not much clear whether these can exert dose-response effects [76].

\section{CONCLUSION AND FUTURE PROSPECTS}

Many studies have confirmed that the quorum quenching based approaches have significant potential as antibacterial agents over the past few years. Several studies signify the use of these methods for the functionalization of medical devices. Thus far, there is limited knowledge about the possible mechanisms of resistance that bacteria could develop in response to the quorum quenching based therapies. The phenomenon of resistance resulting from the evolution in the milieu of selection pressure favoring the progress of resistant strains as have already observed in the case of antibiotics. Although, few quorum sensing inhibitors for instance azithromycin have found to inhibit the growth, many others possess a moderate or quite little effect on growth rate. The quorum quenching enzymes may serve as some ideal contenders with minimal side effects and effective inhibitory activity against the biofilms. However, the quorum-sensing inhibition is still a prospective approach to increase the therapeutic arsenal for the management of bacterial infections in addition to the conventional antimicrobial agents. The comprehensive effect of these strategies on the bacterial physiological properties has shown that quorum interference-based approaches would be 
appropriate for reducing bacterial virulence and restoring the antibiotic resistance through decreasing the biofilm formation and decreasing bacteriophage resistance, therefore, opening the way for imminent combinational therapies. Further studies are desirable to establish the effects induced these strategies for single bacterial species as well as for bacterial communities. The future explorations will direct the extent of the action of quorum sensing interfering molecules and their implications as therapeutic agents, as coating agents for medical implants and as combinational therapeutics.

Table 1. Quorum sensing interference molecules for medical devices

\begin{tabular}{|c|c|c|c|c|}
\hline Devices & Quorum quenching molecules & Strategies & & References \\
\hline Catheters & $\begin{array}{l}\text { Furanones } \\
5 \text { fluorouracil } \\
\text { Acylase (Aspergillus melleus) } \\
\text { Acylase (Aspergillus } \\
\text { melleus) combined with a-amylase (Bacillus } \\
\text { amyloliquefaciens) }\end{array}$ & $\begin{array}{l}\text { Quorum } \\
\text { inhibitor } \\
\text { Quorum } \\
\text { enzymes }\end{array}$ & $\begin{array}{l}\text { sensing } \\
\text { quenching }\end{array}$ & {$[60,61]$} \\
\hline Urinary catheters & Thiazolidinedione-8 & $\begin{array}{l}\text { Quorum } \\
\text { inhibitor }\end{array}$ & sensing & [54] \\
\hline Coatings & $\begin{array}{l}\text { 5-methylene-1-(prop-2-enoyl)-4-(2-fluorophenyl)- } \\
\text { dihydropyrrol-2-one) } \\
\text { PLL SsoPox from Sulfolobus solfataricus }\end{array}$ & $\begin{array}{l}\text { Quorum } \\
\text { inhibitor } \\
\text { Quorum } \\
\text { enzymes }\end{array}$ & $\begin{array}{l}\text { sensing } \\
\text { quenching }\end{array}$ & $\begin{array}{l}{[53]} \\
{[51,65]}\end{array}$ \\
\hline Capsules & Autoinducer-2 processing kinase LsrK & $\begin{array}{l}\text { Quorum } \\
\text { enzymes }\end{array}$ & quenching & [66] \\
\hline $\begin{array}{l}\text { Colonization-resistant } \\
\text { materials }\end{array}$ & Truncated autoinducer peptide-II & Peptides & & [57] \\
\hline $\begin{array}{ll}\text { Implanted medical } \\
\text { devices } \\
\text { Dacron graft }\end{array}$ & $\begin{array}{l}\text { DHP derivatives and furanones } \\
\text { RNA-III inhibiting peptide }\end{array}$ & $\begin{array}{l}\text { Quorum } \\
\text { inhibitor } \\
\text { Peptides }\end{array}$ & sensing & $\begin{array}{l}{[77]} \\
{[59]}\end{array}$ \\
\hline Nanofibers & $\begin{array}{l}\text { Acylase from porcine kidney } \\
\text { Macrocyclic peptides }\end{array}$ & $\begin{array}{l}\text { Quorum } \\
\text { enzymes } \\
\text { Peptides }\end{array}$ & quenching & $\begin{array}{l}{[78]} \\
{[56]}\end{array}$ \\
\hline Topical therapies & Lactonase (Bacillus sp.ZA12) & $\begin{array}{l}\text { Quorum } \\
\text { enzymes }\end{array}$ & quenching & [31] \\
\hline Nano-vectors & Polyphenols of honey & Natural cor & hpounds & [55] \\
\hline Prosthesis & FS3 & Peptides & & [58] \\
\hline
\end{tabular}

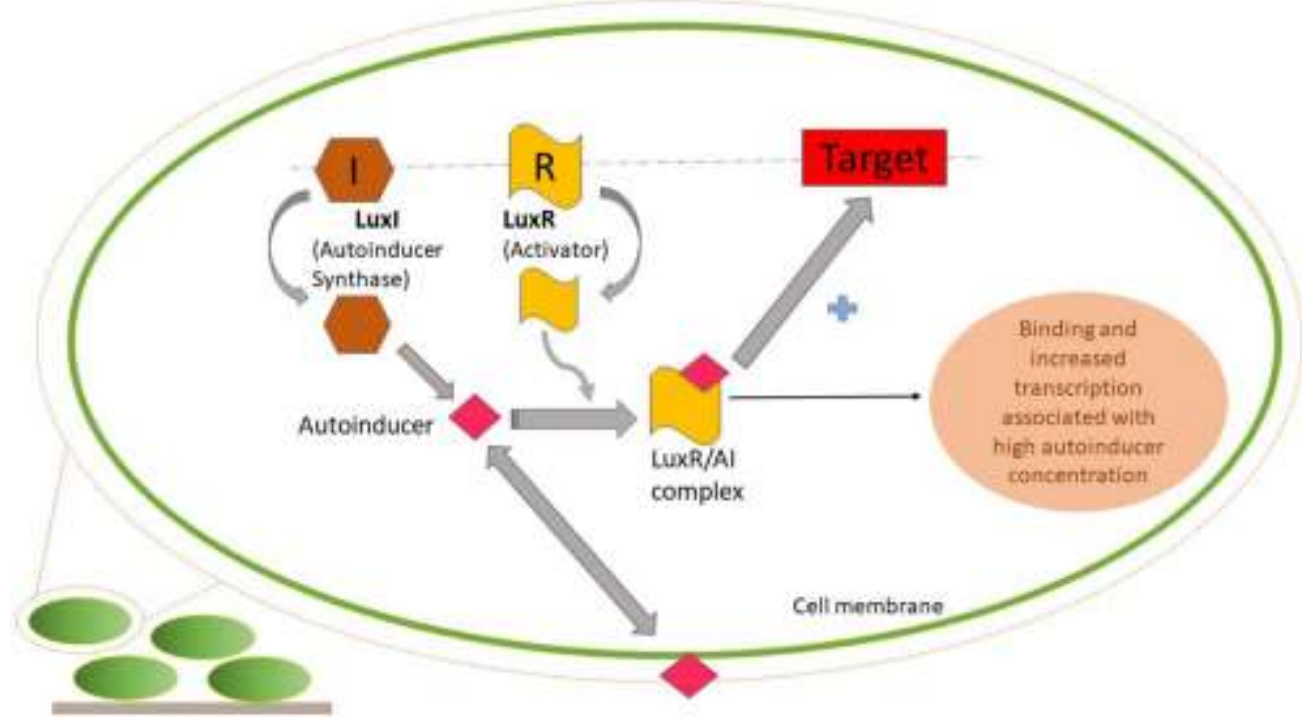

Figure 1. The LuxI, LuxR type quorum sensing. The autoinducer synthase (Luxl-like) catalyzes the synthesis of acylhomoserine lactone (AHL) which can diffuse through the bacterial cell membrane. The transcriptional regulator (LuxR) binds with the diffusing $\mathrm{AHL}$ which can upregulate the transcription of the target genes. 


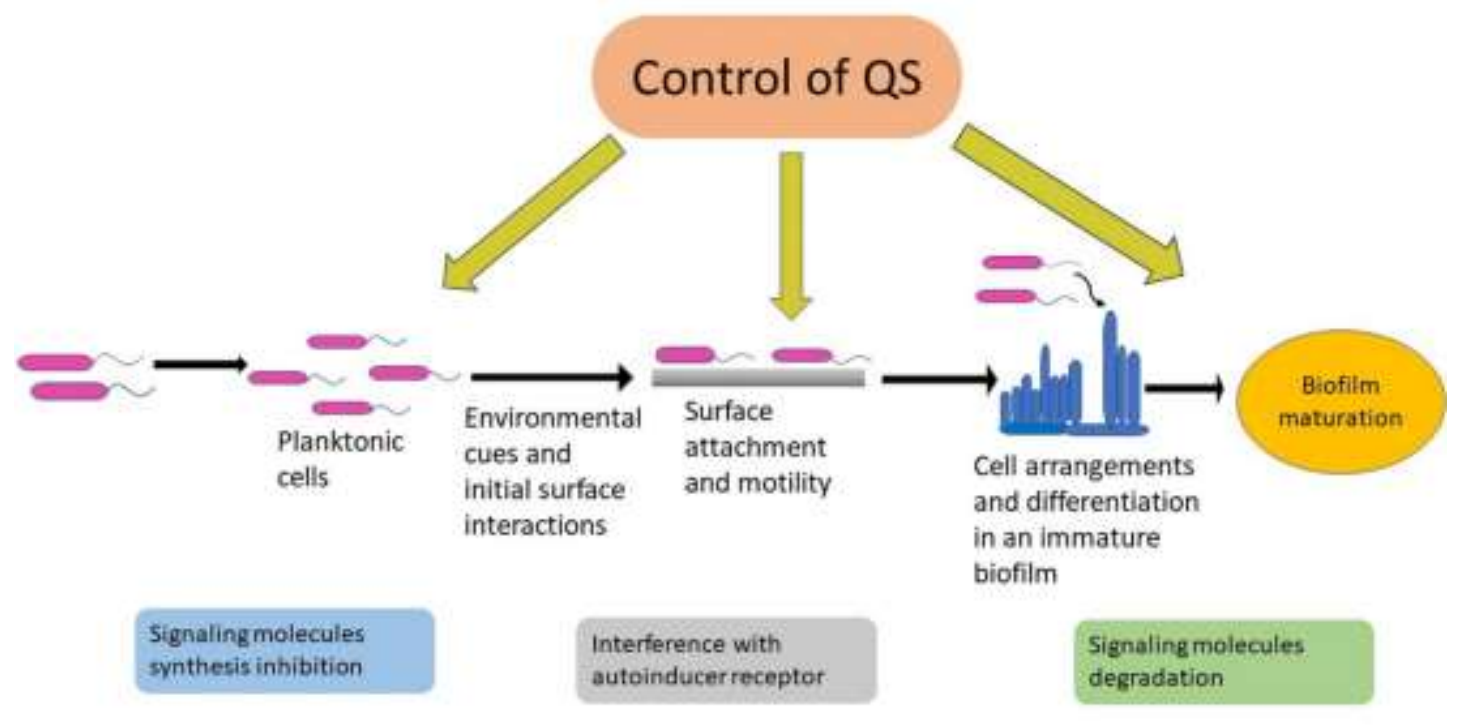

Figure 2. Step involved in biofilm formation and control of quorum sensing for the inhibition of surface colonization.

Funding: This research received no external funding.

Conflicts of Interest: The authors declare no conflict of interest.

\section{REFERENCES}

1 Remy B, Mion S, Plener L, Elias M, Chabriere E, Daude D. Interference in Bacterial Quorum Sensing: A Biopharmaceutical Perspective. Front Pharmacol, 2018; 9: 203.

2 Papenfort K, Bassler BL. Quorum sensing signal-response systems in Gram-negative bacteria. Nat Rev Microbiol, 2016; 14: 576-88.

3 Jiang T, Li M. Quorum sensing inhibitors: a patent review. Expert Opin Ther Pat, 2013; 23: 867-94.

4 Baloch Z, Aslam B, Muzammil S, Khurshid M, Rasool MH, Ma K. Selection inversion: a probable tool against antibiotic resistance. Infect Drug Resist, 2018; 11: 1903-5.

5 Qamar MU, Saleem S, Toleman MA, Saqalein M, Waseem M, Nisar MA, Khurshid M, Taj Z, Jahan S. In vitro and in vivo activity of Manuka honey against NDM-1-producing Klebsiella pneumoniae ST11. Future Microbiol, 2018; 13: 13-26.

6 Akash MSH, Rehman K, Fiayyaz F, Sabir S, Khurshid M. Diabetes-associated infections: development of antimicrobial resistance and possible treatment strategies. Arch Microbiol, 2020.

7 Khurshid M, Rasool MH, Ashfaq UA, Aslam B, Waseem M. Emergence of ISAba1 harboring carbapenemresistant Acinetobacter baumannii isolates in Pakistan. Future Microbiol, 2017; 12: 1261-9.

8 Khan AA, Shrivastava A, Khurshid M. Normal to cancer microbiome transformation and its implication in cancer diagnosis. Biochim Biophys Acta, 2012; 1826: 331-7.

9 Riedel K, Hentzer M, Geisenberger O, Huber B, Steidle A, Wu H, Hoiby N, Givskov M, Molin S, Eberl L. Nacylhomoserine-lactone-mediated communication between Pseudomonas aeruginosa and Burkholderia cepacia in mixed biofilms. Microbiology, 2001; 147: 3249-62.

10 Pacheco AR, Sperandio V. Shiga toxin in enterohemorrhagic E.coli: regulation and novel anti-virulence strategies. Front Cell Infect Microbiol, 2012; 2: 81.

11 Ruffin M, Brochiero E. Repair Process Impairment by Pseudomonas aeruginosa in Epithelial Tissues: Major Features and Potential Therapeutic Avenues. Front Cell Infect Microbiol, 2019; 9: 182.

12 Barr HL, Halliday N, Camara M, Barrett DA, Williams P, Forrester DL, Simms R, Smyth AR, Honeybourne D, Whitehouse JL, Nash EF, Dewar J, Clayton A, Knox AJ, Fogarty AW. Pseudomonas aeruginosa quorum sensing molecules correlate with clinical status in cystic fibrosis. Eur Respir J, 2015; 46: 1046-54.

13 Rampioni G, Leoni L, Williams P. The art of antibacterial warfare: Deception through interference with quorum sensing-mediated communication. Bioorg Chem, 2014; 55: 60-8.

14 Maura D, Ballok AE, Rahme LG. Considerations and caveats in anti-virulence drug development. Curr Opin Microbiol, 2016; 33: 41-6.

15 Wagner S, Sommer R, Hinsberger S, Lu C, Hartmann RW, Empting M, Titz A. Novel Strategies for the Treatment of Pseudomonas aeruginosa Infections. J Med Chem, 2016; 59: 5929-69. 
16 Starkey M, Lepine F, Maura D, Bandyopadhaya A, Lesic B, He J, Kitao T, Righi V, Milot S, Tzika A, Rahme L. Identification of anti-virulence compounds that disrupt quorum-sensing regulated acute and persistent pathogenicity. PLoS Pathog, 2014; 10: e1004321.

17 Pires DP, Melo L, Vilas Boas D, Sillankorva S, Azeredo J. Phage therapy as an alternative or complementary strategy to prevent and control biofilm-related infections. Curr Opin Microbiol, 2017; 39: 48-56.

18 Qin X, Sun Q, Yang B, Pan X, He Y, Yang H. Quorum sensing influences phage infection efficiency via affecting cell population and physiological state. J Basic Microbiol, 2017; 57: 162-70.

19 Hoyland-Kroghsbo NM, Maerkedahl RB, Svenningsen SL. A quorum-sensing-induced bacteriophage defense mechanism. MBio, 2013; 4: e00362-12.

20 Hoque MM, Naser IB, Bari SMN, Zhu J, Mekalanos JJ, Faruque SM. Quorum Regulated Resistance of Vibrio cholerae against Environmental Bacteriophages. Sci Rep, 2016; 6: 37956.

21 Mumford R, Friman VP. Bacterial competition and quorum-sensing signalling shape the eco-evolutionary outcomes of model in vitro phage therapy. Evol Appl, 2017; 10: 161-9.

22 Moker N, Dean CR, Tao J. Pseudomonas aeruginosa increases formation of multidrug-tolerant persister cells in response to quorum-sensing signaling molecules. J Bacteriol, 2010; 192: 1946-55.

23 Maura D, Hazan R, Kitao T, Ballok AE, Rahme LG. Evidence for Direct Control of Virulence and Defense Gene Circuits by the Pseudomonas aeruginosa Quorum Sensing Regulator, MvfR. Sci Rep, 2016; 6: 34083.

24 Liang $\mathrm{H}$, Deng X, Li X, Ye Y, Wu M. Molecular mechanisms of master regulator VqsM mediating quorum-sensing and antibiotic resistance in Pseudomonas aeruginosa. Nucleic Acids Res, 2014; 42: 10307-20.

25 Savage VJ, Chopra I, O'Neill AJ. Staphylococcus aureus biofilms promote horizontal transfer of antibiotic resistance. Antimicrob Agents Chemother, 2013; 57: 1968-70.

26 de la Fuente-Nunez C, Reffuveille F, Fernandez L, Hancock RE. Bacterial biofilm development as a multicellular adaptation: antibiotic resistance and new therapeutic strategies. Curr Opin Microbiol, 2013; 16: 580-9.

27 He X, Lu F, Yuan F, Jiang D, Zhao P, Zhu J, Cheng H, Cao J, Lu G. Biofilm Formation Caused by Clinical Acinetobacter baumannii Isolates Is Associated with Overexpression of the AdeFGH Efflux Pump. Antimicrob Agents Chemother, 2015; 59: 4817-25.

28 Brackman G, Cos P, Maes L, Nelis HJ, Coenye T. Quorum sensing inhibitors increase the susceptibility of bacterial biofilms to antibiotics in vitro and in vivo. Antimicrob Agents Chemother, 2011; 55: 2655-61.

29 Guo Q, Wei Y, Xia B, Jin Y, Liu C, Pan X, Shi J, Zhu F, Li J, Qian L, Liu X, Cheng Z, Jin S, Lin J, Wu W. Identification of a small molecule that simultaneously suppresses virulence and antibiotic resistance of Pseudomonas aeruginosa. Sci Rep, 2016; 6: 19141.

30 Stenvang M, Dueholm MS, Vad BS, Seviour T, Zeng G, Geifman-Shochat S, Sondergaard MT, Christiansen G, Meyer RL, Kjelleberg S, Nielsen PH, Otzen DE. Epigallocatechin Gallate Remodels Overexpressed Functional Amyloids in Pseudomonas aeruginosa and Increases Biofilm Susceptibility to Antibiotic Treatment. J Biol Chem, 2016; 291: 26540-53.

31 Gupta P, Chhibber S, Harjai K. Efficacy of purified lactonase and ciprofloxacin in preventing systemic spread of Pseudomonas aeruginosa in murine burn wound model. Burns, 2015; 41: 153-62.

32 Jiang Q, Chen J, Yang C, Yin Y, Yao K. Quorum Sensing: A Prospective Therapeutic Target for Bacterial Diseases. Biomed Res Int, 2019; 2019: 2015978.

33 Plyuta V, Zaitseva J, Lobakova E, Zagoskina N, Kuznetsov A, Khmel I. Effect of plant phenolic compounds on biofilm formation by Pseudomonas aeruginosa. Apmis, 2013; 121: 1073-81.

34 Adonizio A, Leal SM, Jr., Ausubel FM, Mathee K. Attenuation of Pseudomonas aeruginosa virulence by medicinal plants in a Caenorhabditis elegans model system. J Med Microbiol, 2008; 57: 809-13.

35 Zimmer KR, Macedo AJ, Nicastro GG, Baldini RL, Termignoni C. Egg wax from the cattle tick Rhipicephalus (Boophilus) microplus inhibits Pseudomonas aeruginosa biofilm. Ticks Tick Borne Dis, 2013; 4: 366-76.

36 Darby C, Cosma CL, Thomas JH, Manoil C. Lethal paralysis of Caenorhabditis elegans by Pseudomonas aeruginosa. Proc Natl Acad Sci U S A, 1999; 96: 15202-7.

37 Rasmussen TB, Skindersoe ME, Bjarnsholt T, Phipps RK, Christensen KB, Jensen PO, Andersen JB, Koch B, Larsen TO, Hentzer M, Eberl L, Hoiby N, Givskov M. Identity and effects of quorum-sensing inhibitors produced by Penicillium species. Microbiology, 2005; 151: 1325-40.

38 Papaioannou E, Wahjudi M, Nadal-Jimenez P, Koch G, Setroikromo R, Quax WJ. Quorum-quenching acylase reduces the virulence of Pseudomonas aeruginosa in a Caenorhabditis elegans infection model. Antimicrob Agents Chemother, 2009; 53: 4891-7.

39 Hentzer M, Wu H, Andersen JB, Riedel K, Rasmussen TB, Bagge N, Kumar N, Schembri MA, Song Z, Kristoffersen P, Manefield M, Costerton JW, Molin S, Eberl L, Steinberg P, Kjelleberg S, Hoiby N, Givskov M. Attenuation of Pseudomonas aeruginosa virulence by quorum sensing inhibitors. EMBO J, 2003; 22: 3803-15.

40 Bjarnsholt T, Jensen PO, Rasmussen TB, Christophersen L, Calum H, Hentzer M, Hougen HP, Rygaard J, Moser C, Eberl L, Hoiby N, Givskov M. Garlic blocks quorum sensing and promotes rapid clearing of pulmonary Pseudomonas aeruginosa infections. Microbiology, 2005; 151: 3873-80.

41 Gillaspy AF, Hickmon SG, Skinner RA, Thomas JR, Nelson CL, Smeltzer MS. Role of the accessory gene regulator (agr) in pathogenesis of staphylococcal osteomyelitis. Infect Immun, 1995; 63: 3373-80. 
42 Cady NC, McKean KA, Behnke J, Kubec R, Mosier AP, Kasper SH, Burz DS, Musah RA. Inhibition of biofilm formation, quorum sensing and infection in Pseudomonas aeruginosa by natural products-inspired organosulfur compounds. PLoS One, 2012; 7: e38492.

43 Walz JM, Avelar RL, Longtine KJ, Carter KL, Mermel LA, Heard SO. Anti-infective external coating of central venous catheters: a randomized, noninferiority trial comparing 5-fluorouracil with chlorhexidine/silver sulfadiazine in preventing catheter colonization. Crit Care Med, 2010; 38: 2095-102.

44 van Delden C, Kohler T, Brunner-Ferber F, Francois B, Carlet J, Pechere JC. Azithromycin to prevent Pseudomonas aeruginosa ventilator-associated pneumonia by inhibition of quorum sensing: a randomized controlled trial. Intensive Care Med, 2012; 38: 1118-25.

45 Saiman L, Marshall BC, Mayer-Hamblett N, Burns JL, Quittner AL, Cibene DA, Coquillette S, Fieberg AY, Accurso FJ, Campbell PW, 3rd. Azithromycin in patients with cystic fibrosis chronically infected with Pseudomonas aeruginosa: a randomized controlled trial. Jama, 2003; 290: 1749-56.

46 Tateda K, Comte R, Pechere JC, Kohler T, Yamaguchi K, Van Delden C. Azithromycin inhibits quorum sensing in Pseudomonas aeruginosa. Antimicrob Agents Chemother, 2001; 45: 1930-3.

47 Smyth AR, Cifelli PM, Ortori CA, Righetti K, Lewis S, Erskine P, Holland ED, Givskov M, Williams P, Camara M, Barrett DA, Knox A. Garlic as an inhibitor of Pseudomonas aeruginosa quorum sensing in cystic fibrosis--a pilot randomized controlled trial. Pediatr Pulmonol, 2010; 45: 356-62.

48 Grandclement C, Tannieres M, Morera S, Dessaux Y, Faure D. Quorum quenching: role in nature and applied developments. FEMS Microbiol Rev, 2016; 40: 86-116.

49 Bzdrenga J, Daude D, Remy B, Jacquet P, Plener L, Elias M, Chabriere E. Biotechnological applications of quorum quenching enzymes. Chem Biol Interact, 2017; 267: 104-15.

50 Francolini I, Vuotto C, Piozzi A, Donelli G. Antifouling and antimicrobial biomaterials: an overview. Apmis, 2017; 125: 392-417.

51 Hraiech S, Hiblot J, Lafleur J, Lepidi H, Papazian L, Rolain JM, Raoult D, Elias M, Silby MW, Bzdrenga J, Bregeon $\mathrm{F}$, Chabriere E. Inhaled lactonase reduces Pseudomonas aeruginosa quorum sensing and mortality in rat pneumonia. PLoS One, 2014; 9: e107125.

52 Hume EB, Baveja J, Muir B, Schubert TL, Kumar N, Kjelleberg S, Griesser HJ, Thissen H, Read R, Poole-Warren LA, Schindhelm K, Willcox MD. The control of Staphylococcus epidermidis biofilm formation and in vivo infection rates by covalently bound furanones. Biomaterials, 2004; 25: 5023-30.

53 Ozcelik B, Ho KKK, Glattauer V, Willcox M, Kumar N, Thissen H. Poly(ethylene glycol)-Based Coatings Combining Low-Biofouling and Quorum-Sensing Inhibiting Properties to Reduce Bacterial Colonization. ACS Biomater Sci Eng, 2017; 3: 78-87.

54 Shenderovich J, Feldman M, Kirmayer D, Al-Quntar A, Steinberg D, Lavy E, Friedman M. Local sustained-release delivery systems of the antibiofilm agent thiazolidinedione-8 for prevention of catheter-associated urinary tract infections. Int J Pharm, 2015; 485: 164-70.

55 Prateeksha, Singh BR, Shoeb M, Sharma S, Naqvi AH, Gupta VK, Singh BN. Scaffold of Selenium Nanovectors and Honey Phytochemicals for Inhibition of Pseudomonas aeruginosa Quorum Sensing and Biofilm Formation. Front Cell Infect Microbiol, 2017; 7: 93.

56 Kratochvil MJ, Yang T, Blackwell HE, Lynn DM. Nonwoven Polymer Nanofiber Coatings That Inhibit Quorum Sensing in Staphylococcus aureus: Toward New Nonbactericidal Approaches to Infection Control. ACS Infect Dis, 2017; 3: 271-80.

57 Kim MK, Zhao A, Wang A, Brown ZZ, Muir TW, Stone HA. Surface-attached molecules control Staphylococcus aureus quorum sensing and biofilm development. Nat Microbiol, 2017; 2: 17080.

58 Cirioni O, Mocchegiani F, Cacciatore I, Vecchiet J, Silvestri C, Baldassarre L, Ucciferri C, Orsetti E, Castelli P, Provinciali M, Vivarelli M, Fornasari E, Giacometti A. Quorum sensing inhibitor FS3-coated vascular graft enhances daptomycin efficacy in a rat model of staphylococcal infection. Peptides, 2013; 40: 77-81.

59 Balaban N, Giacometti A, Cirioni O, Gov Y, Ghiselli R, Mocchegiani F, Viticchi C, Del Prete MS, Saba V, Scalise G, Dell'Acqua G. Use of the quorum-sensing inhibitor RNAlll-inhibiting peptide to prevent biofilm formation in vivo by drug-resistant Staphylococcus epidermidis. J Infect Dis, 2003; 187: 625-30.

60 Grover N, Plaks JG, Summers SR, Chado GR, Schurr MJ, Kaar JL. Acylase-containing polyurethane coatings with anti-biofilm activity. Biotechnol Bioeng, 2016; 113: 2535-43.

61 Ivanova K, Fernandes MM, Mendoza E, Tzanov T. Enzyme multilayer coatings inhibit Pseudomonas aeruginosa biofilm formation on urinary catheters. Appl Microbiol Biotechnol, 2015; 99: 4373-85.

62 Ivanova K, Fernandes MM, Francesko A, Mendoza E, Guezguez J, Burnet M, Tzanov T. Quorum-Quenching and Matrix-Degrading Enzymes in Multilayer Coatings Synergistically Prevent Bacterial Biofilm Formation on Urinary Catheters. ACS Appl Mater Interfaces, 2015; 7: 27066-77.

63 Rémy B, Plener L, Poirier L, Elias M, Daudé D, Chabrière E. Harnessing hyperthermostable lactonase from Sulfolobus solfataricus for biotechnological applications. Sci Rep, 2016; 6: 37780.

64 Hiblot J, Gotthard G, Chabriere E, Elias M. Characterisation of the organophosphate hydrolase catalytic activity of SsoPox. Sci Rep, 2012; 2: 779.

65 Guendouze A, Plener L, Bzdrenga J, Jacquet P, Remy B, Elias M, Lavigne JP, Daude D, Chabriere E. Effect of Quorum Quenching Lactonase in Clinical Isolates of Pseudomonas aeruginosa and Comparison with Quorum Sensing Inhibitors. Front Microbiol, 2017; 8: 227. 
66 Rhoads MK, Hauk P, Terrell J, Tsao CY, Oh H, Raghavan SR, Mansy SS, Payne GF, Bentley WE. Incorporating LsrK Al-2 quorum quenching capability in a functionalized biopolymer capsule. Biotechnol Bioeng, 2018; 115: 27889.

67 Kim B, Par KJ, Choi HY, Kwak JH, Kim WG. Differential effects of alkyl gallates on quorum sensing in Pseudomonas aeruginosa. Sci Rep, 2019; 9: 7741.

68 Annapoorani A, Umamageswaran V, Parameswari R, Pandian SK, Ravi AV. Computational discovery of putative quorum sensing inhibitors against LasR and RhIR receptor proteins of Pseudomonas aeruginosa. J Comput Aided Mol Des, 2012; 26: 1067-77.

69 Tan SY, Chua SL, Chen Y, Rice SA, Kjelleberg S, Nielsen TE, Yang L, Givskov M. Identification of five structurally unrelated quorum-sensing inhibitors of Pseudomonas aeruginosa from a natural-derivative database. Antimicrob Agents Chemother, 2013; 57: 5629-41.

70 Yang L, Rybtke MT, Jakobsen TH, Hentzer M, Bjarnsholt T, Givskov M, Tolker-Nielsen T. Computer-aided identification of recognized drugs as Pseudomonas aeruginosa quorum-sensing inhibitors. Antimicrob Agents Chemother, 2009; 53: 2432-43.

71 Schaadt NS, Steinbach A, Hartmann RW, Helms V. Rule-based regulatory and metabolic model for Quorum sensing in P. aeruginosa. BMC Syst Biol, 2013; 7: 81.

72 Leonard PG, Bezar IF, Sidote DJ, Stock AM. Identification of a hydrophobic cleft in the LytTR domain of AgrA as a locus for small molecule interactions that inhibit DNA binding. Biochemistry, 2012; 51: 10035-43.

73 Castillo-Juarez I, Maeda T, Mandujano-Tinoco EA, Tomas M, Perez-Eretza B, Garcia-Contreras SJ, Wood TK, Garcia-Contreras R. Role of quorum sensing in bacterial infections. World J Clin Cases, 2015; 3: 575-98.

74 Guilbault C, Saeed Z, Downey GP, Radzioch D. Cystic fibrosis mouse models. Am J Respir Cell Mol Biol, 2007; 36: 1-7.

75 Hoffmann N, Lee B, Hentzer M, Rasmussen TB, Song Z, Johansen HK, Givskov M, Hoiby N. Azithromycin blocks quorum sensing and alginate polymer formation and increases the sensitivity to serum and stationary-growth-phase killing of Pseudomonas aeruginosa and attenuates chronic P. aeruginosa lung infection in Cftr(-/-) mice. Antimicrob Agents Chemother, 2007; 51: 3677-87.

76 Lesic B, Lepine F, Deziel E, Zhang J, Zhang Q, Padfield K, Castonguay MH, Milot S, Stachel S, Tzika AA, Tompkins RG, Rahme LG. Inhibitors of pathogen intercellular signals as selective anti-infective compounds. PLoS Pathog, 2007; 3: 1229-39.

77 Taunk A, Ho K, Iskander G, Willcox M, Kumar N. Surface immobilization of antibacterial quorum sensing inhibitors by photochemical activation. J Biotechnol Biomater, 2016; 6: 10.4172.

78 Lee J, Lee I, Nam J, Hwang DS. Immobilization and Stabilization of Acylase on Carboxylated Polyaniline Nanofibers for Highly Effective Antifouling Application via Quorum Quenching. ACS Appl Mater Interfaces, 2017; 9: 15424-32.

2020 by the authors. Submitted for possible open access publication under the terms and conditions of the Creative Commons Attribution (CC BY NC) license (https://creativecommons.org/licenses/by-nc/4.0/). 